\section{Effektive Hilfe für Ihre Patientin}

Während der vier bis elf Jahre andauernden Perimenopause leiden viele Frauen unter Menstruationsstörungen, die sich in der Regel als Hypermenorrhö äußern. Hinzu kommen nicht selten restriktive Diäten, eine geringe oder im Gegenteil exzessive körperliche Betätigung, Veränderungen des physischen und psychischen Wohlbefindens, kognitive Einbußen und eine zum Teil erhebliche Müdigkeit.

Etliche dieser Veränderungen können auch auf einen gestörten Eisenhaushalt zurückzuführen sein. Liegt ein Eisenmangel vor, kann dieser nur schwer über die Nahrung bzw. den Verzehr eisenhaltiger Lebensmittel behoben werden. Wenn man sich zu einer medikamentösen Eisensupplementation entschließt, sollte man aus Gründen der Einfachheit orale Präparate bevorzugen, z. B. Eisensulfat als langsam freisetzen- der polymerer Komplex (FSPC) wie in Tardyferon ${ }^{\circledR}$ Depot Retardtabletten.

Die Wirksamkeit von Tardyferon ${ }^{\circledast}$ wurde in der PEARL-Studie belegt, in die Frauen mit symptomatischen Myomen, Hypermenorrhö und einem $\mathrm{Hb}$ Wert $\leq 10,2 \mathrm{~g} / \mathrm{dl}$ aufgenommen wurden. Nach 13 Wochen waren die Blutungen bei $90 \%$ der Patientinnen in der Verumgruppe und bei 19\% der Frauen, die Placebo eingenommen hatte, unter Kontrolle. In der Verumgruppe erreichten die $\mathrm{Hb}$-Spiegel 13,50 g/dl, in der Placebogruppe 12,61 g/dl. Damit konnten die Hb-Spiegel auch bei Frauen mit anhaltender Blutung nahezu normalisiert werden [Donnez J, Tatarchuk TF, Bouchard $\mathrm{P}$ et al. $\mathrm{N}$ Engl J Med. 2012;366: 409-20].

Red.

- Firquet A, Kirschner W, Bitzer J. Gynecol Endocrino 2017;28:1-7

\title{
Thromboseprophylaxe
}

\section{Achten Sie auf die Nierenfunktion!}

_ Für die medikamentöse Prophylaxe venöser Thromboembolien (VTE) bei Patienten mit akuten internistischen Erkrankungen und Bettlägrigkeit sollen nach den Empfehlungen der aktuellen S3Leitlinie vorzugsweise niedermolekulare Heparine (NMH) in einer Hochrisikoprophylaxe-Dosierung oder Fondaparinux verwendet werden. Einfach und unkompliziert sei die Anwendung von Certoparin (Mono-Embolex ${ }^{\circledR}$ ), berichtete Prof. Rupert Bauersachs, Darmstadt. Certoparin ist das das erste und bisher einzige $\mathrm{NMH}$, das unabhängig vom Körpergewicht in fixer Dosierung einmal täglich appliziert wird.

Im klinischen Alltag wird das Thrombosemanagement häufig durch eine eingeschränkte Nierenfunktion und einen dadurch bedingten Anstieg des Blutungs- risikos erschwert. Eine Subgruppenanalyse der CERTIFY-Studie bei 198 Patienten mit schwerer Niereninsuffizienz (glomeruläre Filtrationsrate $\leq 30 \mathrm{ml} / \mathrm{min}$ ) zeigte, dass Certoparin 3.000 I.E. auch bei diesen Patienten bei vergleichbarer Wirksamkeit zu insgesamt signifikant weniger Blutungen und nummerisch weniger schweren Blutungen führte als unfraktioniertes Heparin.

Die VTE-Prophylaxe sollte in der Regel für 6-14 Tage erfolgen. Eine prolongierte bzw. poststationäre Prophylaxe kann im Einzelfall unter Berücksichtigung der individuellen Risikokonstellation erfolgen.

Abdol A. Ameri

- Meet-the-Expert „Verlängerte Thromboseprophylaxe mit Certoparin (Mono-Embolex") - Patientensicherheit im Fokus", 123. Kongress der Deutschen Gesellschaft für Innere Medizin; Mannhein, Mai 2017 (Veranstalter: Aspen)

\section{Dysbalance ausgleichen}

Viele Patienten mit Palpitationen, Tachykardie oder Engegefühl in der Brust klagen auch über Ein- und Durchschlafstörungen. Stress, innere Anspannung sowie eine unrhythmische Lebensführung sind wichtige Ursachen dieser Beschwerden. Die anthroposophische Heilpflanzenkomposition Cardiodoron ${ }^{\circledast}(\mathrm{Zu}-$ bereitung aus Eselsdistel, Frühlingsprimel und Bilsenkraut) kann nicht nur funktionelle Herzbeschwerden lindern, sondern sich auch günstig auf die Schlafqualität auswirken. Dies zeigte sich in einer multizentrischen Beobachtungsstudie. Unter der Therapie mit dem Pflanzen-Trio verbesserten sich sowohl die Ausprägung der Schlafstörungen $(-65 \%)$ als auch der Symptom-Summenscore der Herzbeschwerden (-59\%).

\section{Größter Benefit bei Schichtarbeit}

Auffällig war, dass Patienten, deren Schlafstörungen auf ungewöhnliche Arbeitszeiten zurückzuführen waren, am stärksten von der Therapie profitierten. „Die Unterstützung rhythmischer Prozesse im menschlichen Körper scheint dabei besonders hilfreich zu sein“, erklärte Dr. Claudia Rother, Schwäbisch Gmünd. Wie Andrea Diehl, Saarbrücken, betonte, ist die Phyto-Kombination kein spezifisches Herzmittel, sondern unterstützt die aus der Balance geratenen physiologischen Körperprozesse.

Dr. Martina-Jasmin Utzt

- Weleda-Presseclub: „Anwendung von Cardiodoron ${ }^{\circledR}$ bei Schlafstörungen"; München, März 2017 (Veranstalter: Weleda)

\section{Sie hat etwas für ihr inneres} Gleichgewicht getan!

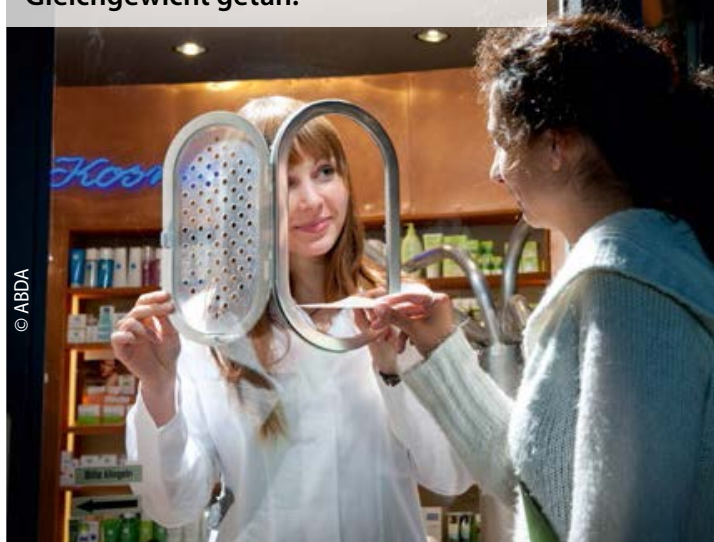

